\title{
Auszeichnung Leitender AOTrauma Chirurg
}

Edgar Mayr

\author{
Auf der Mitgliederversammlung der AOTrauma Deutschland (AOTD) am 9. Mai 2019 \\ in Hamburg wurde - erst zum 3. Mal überhaupt - die Auszeichnung „Leitende/r \\ AOTrauma Chirurg/in am ..." vergeben. Diese wird an besonders qualifizierte \\ Mitglieder der AOTD verliehen, die auf dem Tätigkeitsgebiet der AOTD heraus- \\ ragende Qualifikationen nachgewiesen haben.
}

\section{Ziel der Auszeichnung}

Mit dieser Auszeichnung möchte die AOTD die Sichtbarkeit der ausgezeichneten Person sowie die Qualität und Attraktivität der Einrichtung erhöhen, an der sie tätig ist. Weiterhin soll ein zusätzlicher Anreiz für die Weiterbildung der Mitarbeiter/innen geschaffen werden, insbesondere hinsichtlich der Angebote der AO, um die AOPrinzipien zu lernen, kontinuierlich zu vertiefen und zu verbreiten. Insofern dient die Auszeichnung einerseits der Darstellung der Exzellenz von Mitarbeiter und Klinik anhand von transparenten Kriterien, andererseits dem Ausbau der „AO Schule“. Es handelt sich hierbei nicht um eine personelle oder gar institutionelle Zertifizierung, sondern um eine persönliche Auszeichnung ausschließlich für Mitglieder der AOTD.

\section{Die Geehrten}

Die Auszeichnung erhielten Biberthaler (München), Hartensuer (Münster), A. Hofmann (Kaiserslautern), C. Müller (Karlsruhe), L.-P. Müller (Köln), Schiffer (BergischGladbach) und Rommens (Mainz).

\section{Auswahlkriterien und Deadline für nächste Antragsrunde}

Zur Verleihung der Auszeichnung bedarf es eines Antrages, wozu jedes AOTD-Mitglied berechtigt ist. Die antragstellende Person muss in leitender Funktion an einer herausragenden Klinik mit unfallchirurgischen Schwerpunkt tätig sein. Sie muss Mitglied der Akademie der AOTD sein. Pro Klinik ist nur eine Auszeichnung als „Leitender AOTrauma Chirurg“ möglich.

Voraussetzung für die Zuerkennung der Auszeichnung ist die Erfüllung der vom Erweiterten Präsidium hierzu be-

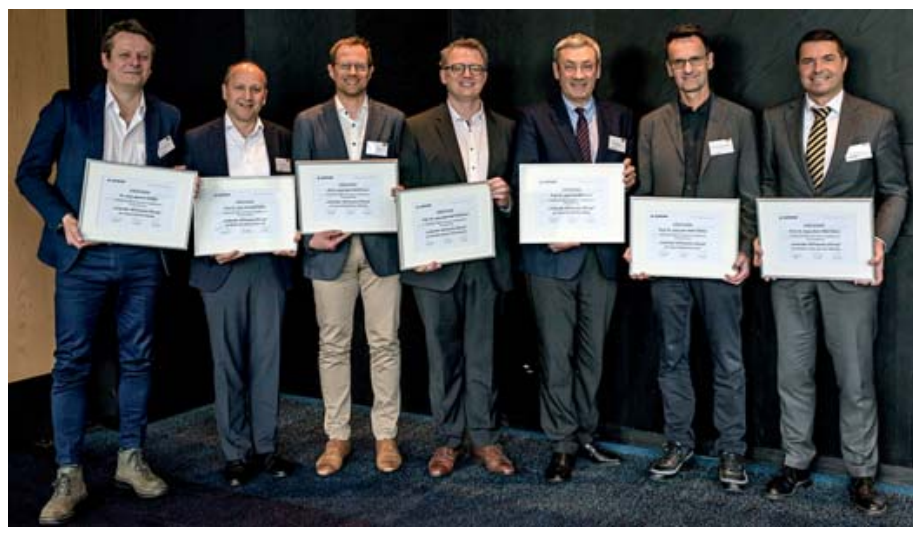

Abb. 1 Die sieben mit der Auszeichnung in Hamburg Geehrten. Quelle: AOTD

schlossenen Kriterien. Die Kriterien und das Antragsformular finden Sie auf der AOTD Homepage oder erhalten Sie über die Geschäftsstelle der AOTD.

Die nächste Verleihung findet während des DKOU 2019 im Oktober statt. Die Deadline für die Einreichung des Antrages ist der 15. September 2019.

Prof. Edgar Mayr, Augsburg

E-Mail: edgar.mayr@klinikum-augsburg.de

Bibliografie

DOI https://doi.org/10.1055/a-0951-3648

OP-JOURNAL 2019; 35: 197 @ Georg Thieme Verlag KG Stuttgart · New York ISSN 0178-1715 